\title{
ACTIVE CHAT MONITORING AND SUSPICIOUS DETECTION OVER INTERNET
}

\author{
Avaghade S.B., Awale Vinayak Rajkumar, Patil Jaydeep Jayvant, Sonavane Sayali Prithviraj, Patil Aishwarya Dilip \\ Department of CSE \\ DACOE, Karad
}

\begin{abstract}
The increasing use of Instant chat messengers to share information, suspicious activities have also increased. There are many sources to share information but instant chat messengers and social networking websites are the quick and easy means to share anything. Sometimes even new stories initially broken up on social media site and further on chat messenger instead of any news channel and newspaper etc. Due to these technology advancements, some people are misusing these instant chat messenger to share suspicious chat activities and make a plan to do something suspicious. This kind is mainly available in texture in the format. With the advancement of internet technology and the change in mood of communication and it is found that much first-hand news has been discussed in internet forums well before they are reported in traditional mass media. Also, this communication channel provides an effective channel for illegal activities such as broadcasting of copyrighted movies, threatening messages and online gambling etc. Our proposed threatening messages and online gambling etc. Our proposed system will analyses online plain text sources from selected discussion forums and will classify the text into different groups the text into different groups and system will decide which post is legal and illegal.
\end{abstract}

Keywords - Suspicious activities, Misuse of technology, Classifying the text.

\section{INTRODUCTION}

Chat refers to the process of communicating, interacting and/or exchanging messages over the Internet. It involves two or more individuals that communicate through a chat-enabled service or software. Chat may be delivered through text, verbal, communication. Chat is also known as chatting, online chat or Internet chat. Terrorist activities communicate over application and chat programs over the internet. It also uses these chat applications over the internet for getting their message to younger generation and making all of types terrorists. The chat monitor system is an Important application that could allow for secure chats along with terrorism related chat detection that helps track down spread of terrorist networks and locate the activities using IP addresses. The internet chat application is a dedicated chat application for free internet chat as well as tracking down on spread of terrorism online. Communication provides effective areas for illegal activities such as threatening messages. This system we have created called as Active Chat Monitoring \& Suspicious Chat Detection over Internet which will tackle with these issues. Internet technology had been increasing more. The law looking for solutions to detect these discussion forums for all possible criminal activities and download suspected Postings as evidence for investigation. Active Chat Monitoring System which will tackle with this problem. It has used a data mining algorithm to detect criminal activities, legal and illegal postings. In this system will use text data mining technique. Active Chat Monitoring System will let us help to analyses online plain text sources from selected discussion forums and will classify the text into groups and system will decide which post is legal and illegal accordingly to their points. It will help us to reduce and minimize many criminal activities which are held on social-site such as Facebook, Twitter, Tinder, etc.

\section{EXISTING SYSTEM}

Online chat might allude to any sort of correspondence over the Internet that offers a continuous transmission of instant messages from sender to beneficiary. Chat messages are by and large short keeping in mind the end goal to empower different members to react rapidly. Along these lines, an inclination like a talked discussion is made, which recognizes chatting from other content based online correspondence structures, for example, Internet gatherings and email. Online chat might deliver point-to-point correspondences and in addition multicast interchanges from one sender to numerous collectors and voice and video chat, or might be a component of a web conferencing administration. As information goes through server it constantly Filters it for any suspicious watchwords. The customary examination of Internet chat room dialogs puts an asset trouble on the knowledge group due to the time required to screen a huge number of persistent chat sessions.

\section{PROPOSED SYSTEM}

The proposed System will analyse online plain text sources from selected discussion forums and will classify the text into different groups and system will decide which post is legal and illegal. This system will ensure that the admin may not 
watch all the chat at a time, so in order to stop chatting illegally, the keywords are set by the other person.

- The system to be developed here is a chat facility.

- It is a client-server system with centralized database server.

- There are two-way communications between the client and the server.

- This chat application can be used for group discussion.

- It allows the user to send feedback to the admin.

\section{A. SYSTEM BLOCK DIAGRAM}

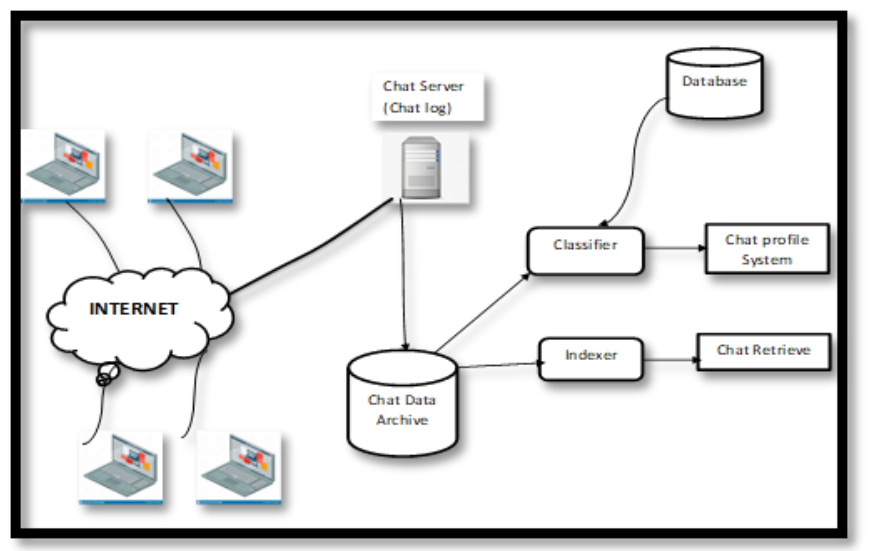

System Architecture

B. Modules

1.Registration Module
2. Chat Room
3.Administrator
4. Keyword
5.Database

1.Registration module

The new user will get registered by using Adhar number. Then user able to use chat room.

\section{Chatroom}

Chat room is to share information via text with a group of other users. Generally speaking, the ability to converse with multiple people in the same conversation differentiates chat rooms from instant messaging programs, which are more typically designed for one-to-one communication.

\section{Administrator}

Admin is responsible to input suspicious keywords into the system to catch the illegal activity over the web.

\section{Keyword}

Keywords are which is set by the admin for chatting purpose. However, the keywords are set by the admin during a chat with the user while using those keywords an error message or an inappropriate message is been viewed to the user at another end

\section{CONCLUSION}

By this work, Active chat monitoring and suspicious chat detection over internet we conclude that using a chat for inappropriate conversation provide a secure access over internet without any further monitoring process. This could be further developed into two user communication with the help of server control. Overall process of Active chat monitoring and suspicious chat detection over internet is done the process helps the people. This can be assured from the above analysis and works. If the given future enhancements are implemented in a correct manner then it can be extending the success of this project in the future. The Project titled Active chat monitoring and suspicious chat detection over internet is tested with sample data and found to be working well. The system has been developed for the users/people. The database approach of developing the system has helped in reducing redundancy of data and improve in the consistency of data in the system. This system is flexible, user friendly.

\section{REFERENCES}

1. Rob Kavet and Gabor Kenzo, "A Perspective on Chat Associated with Suspecious Chat Technology", published by IEEE in 2010 .

2. David W. Cheung, and et al., "Maintenance of discovered association rules in largedatabases: an incremental updating technique," published by IEEE in 1996.

3. Shakil Ahmed, Frans Coenen, and Paul Leng "Treebased Partitioning of Data for Association Rule Mining", published by IEEE in 2012.

4. Daya C. Wimalasuriya and Dejing Dou," OntologyBased Information Extraction: An Introduction and a Survey of Approaches", Journal of Information Science, Volume 36, No. 3, pp. 306-323, 2010.

5. Hosseinkhani, Javad, Mohammad Koochakzaei, Solmaz Keikhaee, and Javid Hosseinkhani Naniz. "Detecting suspicion information on the Web using crime data mining techniques." published by IJARCS in 2015.

6. Alami, Salim, and Omar EL Beqqali. "Detecting Suspicious Profiles Using Text Analysis Within Social Media.," published by JATIT in 2015.

7. Ali, Mohammed Mahmood, Khaja Moizuddin Mohammed, and Lakshmi Rajamani. "Framework for surveillance of instant messages in instant messengers and social neworking sites using data mining and ontology," published by IEEE in 2014. 
8. Harsh Arora and Govind Murari Upadhyay," A Framework for the Detection of Suspicious Discussion on Online Forums using Integrated approach of Support Vector Machine and Particle Swarm Optimization", published by IJARCS in 2015.

9. Placida Tellis, N. Deepika "Expert System to Detect Suspicious Words in Online Messages for Intelligence Agency Using FP-growth Algorithm," published by IJCSMC in 2015.

10. Michael Robertson, Yin Pan, and Bo Yuan, "A Social Approach to Security: Using Social Networks to help detect malicious web content," published by IEEE in 2010.

11. Roger D. Peng "Overview and History of R" Computing for Data Analysis course, Johns Hopkins University

12. N. Pendar, "Toward spotting the pedophile telling victimfrom predator in text chats," IEEE Internet Computing, pp.235-241, 2007.

13. ASERL (Association of Southeastern Research Libraries). 2001. ASERL Virtual Reference Project. [Online]. Available:

\{http://www.aserl.org/projects/vref/default.html\}

[September 21, 2001]. 